\title{
Study the expense of rang and relative rang to carbon ions and oxygen ions among the various elements
}

\author{
Iman Hmood Abdullah*, Inaam Hani Kadhim \\ Department of Physics, College of Education for Pure Sciences, University of Babylon \\ *Corresponding author_E-mail: anaam_hani@yahoo.com
}

Copyright $\odot 2014$ Abdullah and Kadhim. This is an open access article distributed under the Creative Commons Attribution License, which permits unrestricted use, distribution, and reproduction in any medium, provided the original work is properly cited.

\begin{abstract}
In this research study theory to calculate the range of ions of carbon ions of oxygen with atomic number ( 6.8 ) during the passage of the media watchdog of the elements ( lanthanum La, Samarium Sm, Alerdiom Er, Altantalm Ta, gold Au, Lead $\mathrm{Pb}$ and uranium $\mathrm{U})$ with numbers Atomic $(57,62,68,73,82,92)$ the results showed that the Rang of carbon ions and oxygen ions in these circles depends on Quicken goals Atomic exponentially in addition, we find that the term proportional directly proportional to the charged particle energy as well as its dependence on shipment The mass and density of the charged particle. The curves showed that the Rang of oxygen and carbon ions increases with increasing energy, with a note that the Rang of carbon is greater than the Rang of oxygen because the term is inversely proportional to the missile shipment.

As for the term relative lost by each of the ions of oxygen and carbon in target (IAEA) (La, Sm, Er, Ta, Au, $\mathrm{Pb}$, and U). , And compared the results obtained to the Rang with the results of the program SRIM2003 Tests showed good agreement with the use of the equation, especially due to the difficulty in obtaining results of the process over. A computer program has been adopted in the Matlab programming language equations for calculating the range to get the desired results and that is described as graphs.
\end{abstract}

Keywords: Alpha Particles, Matlab, Atomic, Energy, the Program SRIM2003.

\section{Introduction}

The distance traveled by the particle -charged through the material so Evkdtagueth entirely before he got to sleep know the Rang (Rang) and the distance traveled by the particle along this path know the path length overall (Total path length) [1] to calculate the length of the path the total charged particle in the center with a primary energy $\left(\mathrm{E}_{0}\right)$ through to the sleep state

$(\mathrm{E}=0)$ using the following equation [2]

$R(T)=\int_{0}^{T}\left[-\frac{d E}{d x}\right]^{-1} d E$

Where: $\mathrm{R}$ represents the Rang of the charged particle.

Represents the stopping power of the charged particle.

$R=\int_{E}^{0} d x=\int_{E}^{0} \frac{d E}{d E} d x=\int_{0}^{E} \frac{1}{d E / d x} d E=\int_{0}^{E} \frac{d E}{S . N}$

The term is defined in terms of the ability of suspension follows [3]:

Makes a theoretical calculation of the Rang of a very difficult task [4], [5], so a number of scientists have gone to the experimental equations to calculate that amount and Adloha on the basis of their findings.

Bragg and Kleeman [6] gave a formula to calculate the Rang of the grave in the middle of their range if it is known in the midst of another: 
$\frac{R_{1}}{R_{2}}=\frac{\rho_{2}}{\rho_{1}}\left[\frac{A_{1}}{A_{2}}\right]^{1 / 2}$

The $\rho_{1}, \rho_{2}$ represent the density of the middle first, second and $\mathrm{A}_{1}, \mathrm{~A}_{2}$ mass number of materials. But at the same speed Gesimitan Mahontin primary $\beta$, the ratio between the Mdyatea is simply [1]:

$\frac{R_{1}(\beta)}{R_{2}(\beta)}=\frac{Z_{2}^{2} M_{1}}{Z_{1}^{2} M_{2}}$

\section{Factors influencing the term (R) Factors which affect rang}

There are several factors that have a significant impact in the long [7], [8]

i. The Energy

The ability of the stop (s) is inversely proportional to the energy (E) through clarified in beta Bloch equation and including the range $(\mathrm{R})$ is inversely proportional to $(\mathrm{S})$ so it is directly proportional to the proportional energy .

ii. Mass

The small mass of the charged particle at the center of the break for the material will increase the Rang of breaching Article winding paths heightened mass will be less term as is the case for heavy -charged particle.

iii. charge

The ability of suspension increases with increasing particle charge and including the term commensurate with the ability to stop it commensurate with the Rang to box shipment suspension.

iv. Density

The ability of suspension increases with increasing density if term is inversely proportional to the density of the center absorbed.

\section{Results and discussion}

i. calculate the rang of oxygen and carbon ions in atomic goals

Calculation of Range for Oxjeen and Carbon in Atomic Target

According to long- ions of oxygen and carbon in atomic goals ( $\mathrm{La}, \mathrm{Sm}, \mathrm{Er}, \mathrm{Ta}, \mathrm{Au}, \mathrm{Pb}, \mathrm{U}$ ) within the limits of the Rang of the energy $(0.3-100 \mathrm{MeV})$ first of the equation [9]

$R\left(\mathrm{mgcm}^{-2}\right)=0.173 E^{3 / 2} A^{1 / 3}$

It was then replaced in the equation [10]

$R_{1}(\beta)=\left(Z_{2}^{2} M_{1} / Z_{1}^{2} M_{2}\right) R_{2}(\beta)$

According to the term and draw as a function of energy using the program in a language of Matlab and shown in Figures (1) and (2) Apennan over all of the oxygen and carbon as it showed that the curves that the Rang of ions of oxygen and carbon increases with increasing energy, with a note that the Rang of carbon is greater than the Rang of oxygen because term is inversely proportional to the shell shipment.

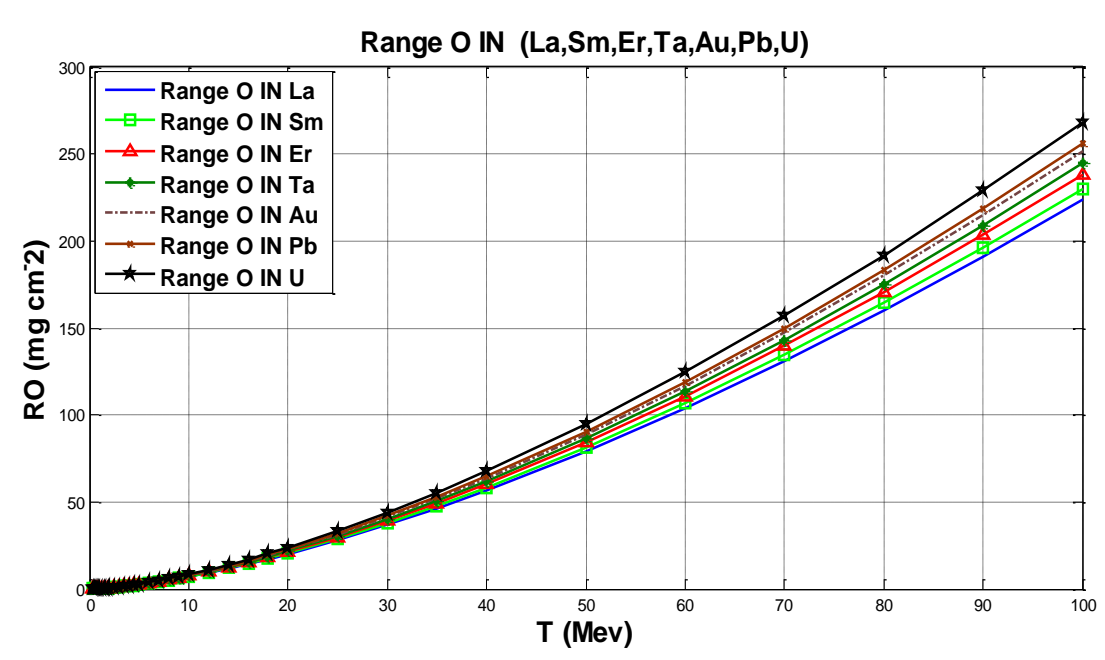

Fig. 1: The Rang of Oxygen Ions Falling on Atomic Targets (La, Sm, Er, Ta, Au, Pb, U) 


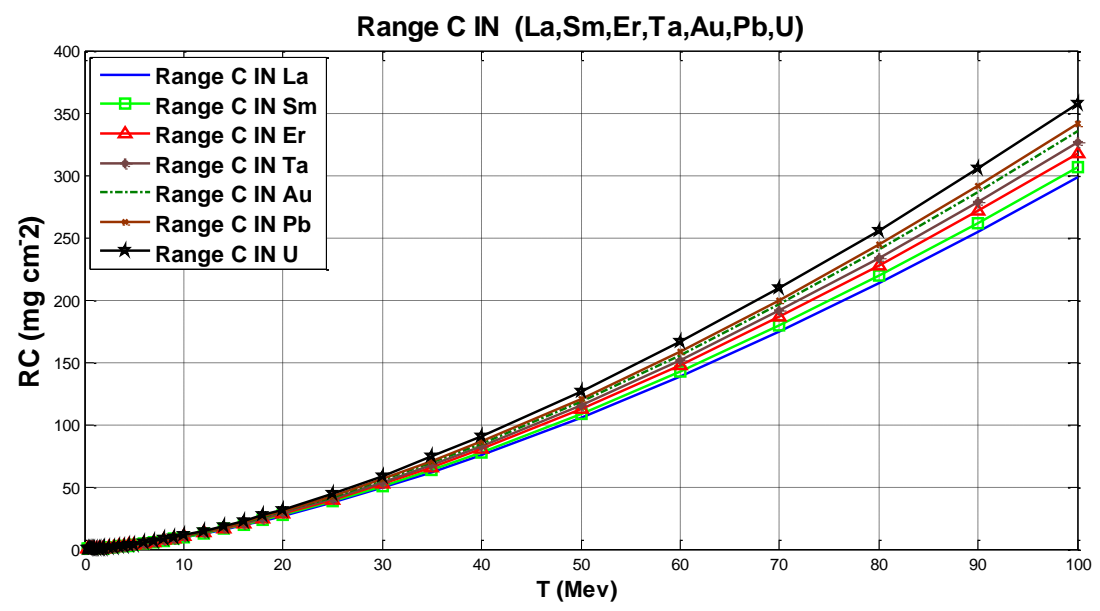

Fig. 2: The Rang of Carbon Ions Falling on Atomic Targets ( $\mathrm{La}, \mathrm{Sm}, \mathrm{Er}, \mathrm{Ta}, \mathrm{Au}, \mathrm{Pb}, \mathrm{U})$

\section{ii. Calculation of relative range for oxjeen and carbon in atomic target}

Have been calculated relative term for carbon ions and oxygen ions in circles Atomic ( $\mathrm{La}, \mathrm{Sm}, \mathrm{Er}, \mathrm{Ta}, \mathrm{Au}, \mathrm{Pb}, \mathrm{U}) \mathrm{using}$ the following equation [10].

The Rang of the energy (0.3-100Mev) and the process took account term relative and drawn as a function of the energy of these ions using the program in a language the Matlab (Figures 3\&4) which illustrate the range relative to ions of oxygen and carbon as a function of energy gross Fallen (ions of oxygen and carbon) on the sequence and shows that the term relative to those ions inversely proportional to the energy of gross Fallen higher the energy of the incident ion, the relative term at least, and this behavior appeared for each of the ions of oxygen and carbon pedestrians in those circles (IAEA).

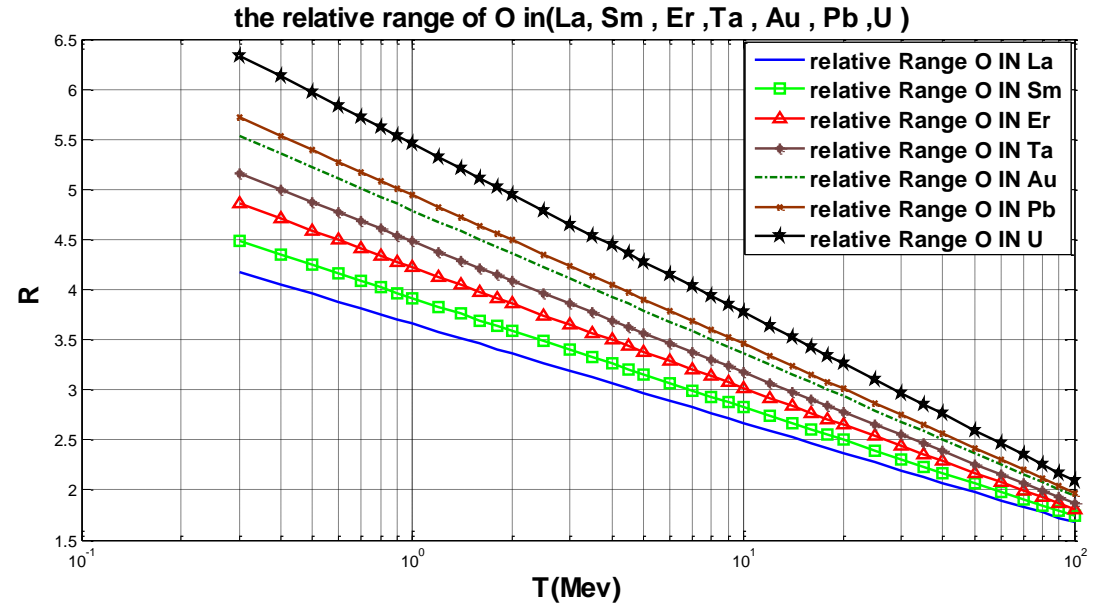

Fig. 3: The Rang Relative to Oxygen Ions Falling on Atomic Goals ( $\mathrm{La}, \mathrm{Sm}, \mathrm{Er}, \mathrm{Ta}, \mathrm{Au}, \mathrm{Pb}, \mathrm{U}$ )

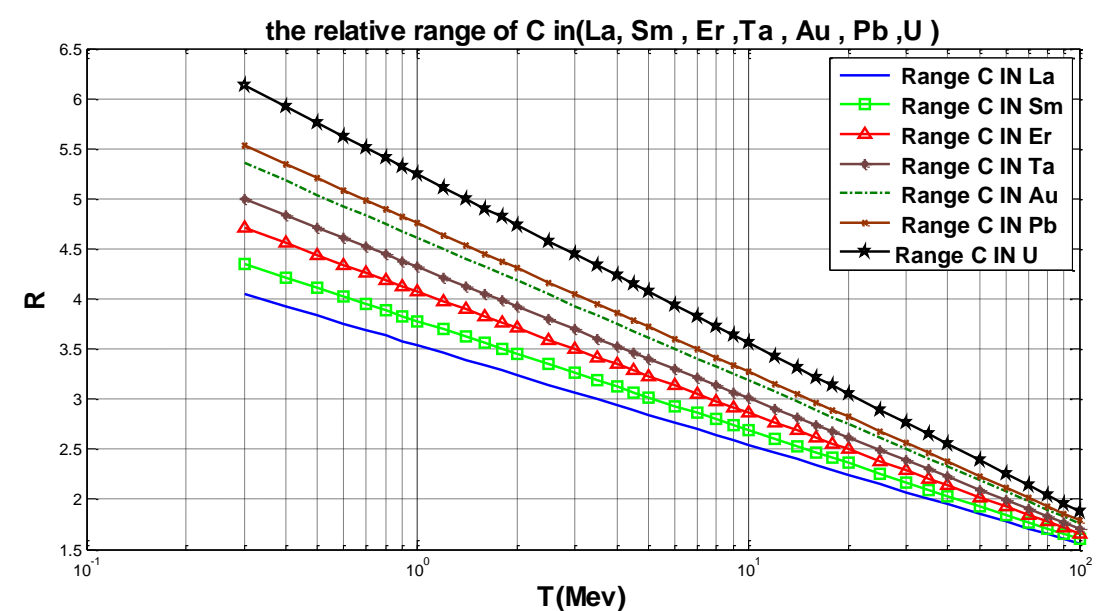

Fig. 4: The Rang Relative to Carbon Ions Falling on Atomic Goals ( $\mathrm{La}, \mathrm{Sm}, \mathrm{Er}, \mathrm{Ta}, \mathrm{Au}, \mathrm{Pb}, \mathrm{U}$ ) 


\section{Conclusion}

The general formula to calculate the range (1) make a theoretical calculation of the Rang of a very difficult task because it is not accurate to calculate the term when the energies of low-lying, so we adopted the equation (6) to calculate the term for greater accuracy in the calculation of the range. From our study of the Rang generally turned out in large influenced by many factors, including the mass of the particle mass -charged heavy impact on the Rang to penetrate the center of the small Vaketlh charged particle at the center of the unshielded material increase over the break of the material, but winding paths. But note that the increase in mass -term at least. And energy when he was stopping ability is inversely proportional to the energy and including the term is inversely proportional to the ability of the stop clear term fit directly proportional to the energy and show us that in the shells of carbon ions and oxygen ions among the pure elements, as well as vehicles. As for the intensity of this factor turned out to big an impact in our calculations of the theoretical capacity of the stop and the Rang of the charged particles (ions of oxygen, carbon ions) in both water and Almaalr and Kabton prominently taking the ability of suspension increases with increasing density. So term is inversely proportional to the density of the middle absorbent. Finally Vqdugdna The shipment of the consignment big role in the calculation of both the ability and the long suspension when he was stopping ability increases with increasing particle charge and the Rang inversely proportional to the Rang to the ability of the stop so is inversely proportional to the square of the shipment

\section{References}

[1] Z.Jianhue, L.Jiarui and L.yi, "Calculations of Nucler Stopping Power and Ranges in the Low- Energy Region ", Chinese Phys .Lett. Vol.6, No.5201-205, (1989).

[2] H.Bichsel, "Stopping Powr and Ranges of Fast Ions in Heavy Elements", Phys. Rev. A, Vol.46, No.95761-5772, (1992).

[3] S.A.Aziz, "Chemical Effect on Bethe - Bloch Stopping Formula", M.Sc. Thesis, Al- Mustansiriyah University 1-57, (2000).

[4] N. Jasim AL - Obiady," Kintetic, Free Electron Gas and Harmonic Oscillator Theories Of Particle Stopping In Medium", M.Sc. Thesis, AL Mustansiryah University, 1-15, (2001).

[5] J.Petola, "Stopping Power for Ions and Clusters In Crystalline Solids", University of Halsinki, Report Series In Physics, HU-P-D108, 8-14, (2003).

[6] A.Sharma etal, "Stopping of Swift Ions in Compounds", Preprint Submitted to Nucl. Instr. and Math.B 1-3, (2003).

[7] O.Kabadayi, "Range Of Medium and High Energy Protons and Alpha Particles in NaI Scintillator", Acta Physica Polonica B, Vol.37, No.61847, (2006)

[8] Y. Zhang, I.Bae, K.Sun, C.Wang, M.Ishimaru, Z.Zhu, W.Jiang and W. Weber," Damage Profile and Ion Distribution of Slow Heavy Ions In Compounds", J.Appl.Phy.105, 1049011-12, (2009). http://dx.doi.org/10.1063/1.3118582

[9] J. F. Zigler, J .P .Biersack [SRIM .com] Contribution by M .Ziegler, D. J .Marwick, G. A. Cuomo, W. A. Porter, S. A. Harrison [SRIM 2003.12]

[10] M. Annunziata, "Nuclear Radiation, Its Interaction With Matter an Radioisotope Decay", 8-14, (2003). 